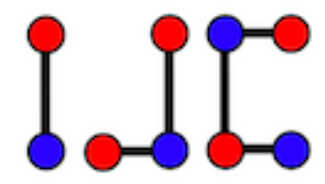

\title{
Size multipartite Ramsey numbers for small paths versus books
}

\author{
Chula Jayawardene ${ }^{\mathrm{a}}$, Jayampathy Ratnayake ${ }^{\mathrm{b}}$ \\ ${ }^{a}$ Department of Mathematics, University of Colombo, Colombo, Sri Lanka \\ ${ }^{b}$ Faculty of Engineering, Sri Lanka Institute of Information Technology, Malambe, Sri Lanka \\ c_jayawardene@yahoo.com, jayampathy.r@sliit.lk
}

\begin{abstract}
Given $j \geq 2$, for graphs $G$ and $H$, the size Ramsey multipartite number $m_{j}(G, H)$ is defined as the smallest natural number $t$ such that any blue red coloring of the edges of the graph $K_{j \times t}$, necessarily containes a red $G$ or a blue $H$ as subgraphs. Let the book with $n$ pages is defined as the graph $K_{1}+K_{1, n}$ and denoted by $B_{n}$. In this paper, we obtain the exact values of the size Ramsey numbers $m_{j}\left(P_{3}, H\right)$ for $j \geq 3$ where $H$ is a book $B_{n}$. We also derive some upper and lower bounds for the size Ramsey numbers $m_{j}\left(P_{4}, H\right)$ where $H$ is a book $B_{n}$.
\end{abstract}

Keywords: Graph theory, Ramsey Theory

Mathematics Subject Classification : 05C55, 05D10

\section{Introduction}

Throughout this paper, all graphs $G=(V, E)$ considered are finite simple(without loops and multiple edges) graphs. The neighborhood of a vertex $v \in V$ is defined as the set of vertices adjacent to $v$ and is denoted by $N(v)$. The degree of $v, d(v)$, is defined as the cardinality of $N(v)$, i.e. $d(v)=|N(v)|$. The complete graph on $n$ vertices is denoted by $K_{n}$. Also let the graph $K_{j, s}$ represents complete bipartite graph with all the partite sets(namely $j$ of them) having exactly $s$ vertices in each partite set. Finally, the book with $n$ pages is defined as the graph $K_{1}+K_{1, n}$ and denoted by $B_{n}$. Note that $B_{n}$ can be thought of as $n$ triangles with one common edge.

Let $G$ and $H$ be two graphs. If for every two coloring (red and blue) of the edges of a complete graph $K_{n}$, there exists a copy of $G$ in the first color (red) or a copy of $H$ in the second color

Received: 30 July 2015, Revised: 26 August 2016, Accepted: 22 September 2016. 
(blue), we say $K_{n} \rightarrow(G, H)$. The classical Ramsey number $r(n, m)$ is defined as the smallest positive integer $s$ such that $K_{s} \rightarrow\left(K_{n}, K_{m}\right)$. The exact determination of these numbers has been studied in detail up to $r(5,5)$ (see [5] for a survey). However, progress has stalled at present in $r(5,5)$ which has been shown to be one on the numbers $\{43,44, \ldots, 49\}$. One of the main variations of the classical Ramsey numbers is the concept of size Ramsey numbers introduced by Erdös, Faudree, Rousseau and Shelph [3], [1], [4], [6] and [10]. Another offshoot of Ramsey numbers and of the size Ramsey numbers, is the notion of size multipartite Ramsey numbers were introduced by Burger and Vuuren [2] and later developed by Baskoro et al and [7]. More precisely, given $j \geq 2$, for graphs $G$ and $H$, the size Ramsey multipartite number $m_{j}(G, H)$ is defined as the smallest natural number $t$ such that any blue red coloring of the edges of the graph $K_{j \times t}$, necessarily contains a red $G$ or a blue $H$ as subgraphs. Up to now a few classes of such Ramsey Numbers have been investigated by Syafrizal Sy, Baskaro et al. (see [8] and [9]). In this paper, motivated by the work done by Rousseau et al. on Book Ramsey numbers we try to investigate the nature of size Ramsey multipartite numbers for small paths verses books.

\section{Size Ramsey numbers related to paths of size three verses Books}

In this section we obtain exact values for size Ramsey numbers $m_{j}\left(P_{3}, B_{n}\right)$, where $j \geq 3$ and $n \geq 1$. Our main results for this section are given in Theorems 1.1 and 1.2.

Theorem 1.1. If $j \geq n-3$, then

$$
m_{j}\left(P_{3}, B_{n}\right)= \begin{cases}2 & \text { if } j=n+3, \text { and } j \text { is even } \\ 1 & \text { otherwise }\end{cases}
$$

The proof of this theorem is left to the reader. Henceforth, for all results and definitions in this section, we will assume that $j<n-3$.

Theorem 1.2. If $j<n-3$, then

$$
m_{j}\left(P_{3}, B_{n}\right)= \begin{cases}\left\lceil\frac{n}{j-2}\right\rceil & \text { if } s>j-1, \text { where } s=\left\lceil\frac{n}{j-2}\right\rceil \\ \left\lceil\frac{n+1}{j-2}\right\rceil & \text { otherwise }\end{cases}
$$

This theorem is a direct consequence of Propositions 1.4 and 1.5. We begin by proving the following proposition. 
Proposition 1.1. $m_{j}\left(P_{3}, B_{n}\right) \geq\left\lceil\frac{n}{j-2}\right\rceil$ when $j<n-3$.

Proof. Consider the graph $K_{j \times s}$, where $s=\left\lceil\frac{n}{j-2}\right\rceil-1$, such that all the edges are colored blue. Then, the graph has no red $P_{3}$. Moreover, if $B_{m}$ is a blue book contained in the graph, then

$$
m \leq(j-2) s<\left(\frac{n}{j-2}+1\right)(j-2)-(j-2)=n .
$$

Therefore, the graph contains no blue $B_{n}$. Hence $m_{j}\left(P_{3}, B_{n}\right) \geq\left\lceil\frac{n}{j-2}\right\rceil$.

Proposition 1.2. $m_{j}\left(P_{3}, B_{n}\right) \leq\left\lceil\frac{n+1}{j-2}\right\rceil$ when $j<n-3$.

Proof. Note that, in this case $s \geq 2$. Consider a red $P_{3}$-free red and blue coloring of $K_{j \times s}$, where $s=\left\lceil\frac{n+1}{j-2}\right\rceil$. Let $H_{R}$ and $H_{B}$ be the red and blue subgraphs of $G$ respectively, induced by the coloring. Thus $G=H_{R} \oplus H_{B}$. Then $H_{R}$ has no $P_{3}$ and hence is a matching with at most $\left\lfloor\frac{s j}{2}\right\rfloor$ edges. Therefore, $H_{R}$ must satisfy one of the following cases.

Case 1: There is an isolated vertex $u$ in partite set $A$.

With out loss of generality, choose an edge $e=v_{1} v_{2}$ in $H_{R}$ between partite sets $B$ and $C$, where $v_{1} \in B, v_{2} \in C$ and $B \neq A$. This forces a book $B_{m}$ in $H_{B}$, where

$$
m=(j-2) s-1=(j-2)\left\lceil\frac{n+1}{j-2}\right\rceil-1 \geq n,
$$

with the highest degree vertices of $B_{m}$ chosen to be $u$ and $v_{1}$.

Case 2: There exists a pair of edges, $e_{1}=v_{1} v_{2}$ and $e_{2}=u_{1} u_{2}$, between two or three distinct partite sets.

That is, there are two distinct partite sets $A$ and $B$, such that $v_{1}, u_{1} \in A$ and $v_{2} \in B$.

This forces a book $B_{m}$ in $H_{B}$, where $m \geq(j-2) s-1=(j-2)\left\lceil\frac{n+1}{j-2}\right\rceil-1 \geq n$, with the highest degree vertices of $B_{m}$ chosen to be $u_{1}$ and $v_{2}$.

Thus, there is always a $B_{n}$ in $H_{B}$ as required. 


\section{Bad colorings - Type 1 and Type 2}

Definitions of Type 1 and Type 2 red and blue bad colorings of $K_{r \times t}\left(=H_{R} \oplus H_{B}\right)$ are given below.

- Type 1: $H_{R}$ consists of a perfect matching with every pair of edges is incident to at least three partite sets

- Type 2: $H_{R}$ consists of a partite set, $A$, consisting of only isolated vertices and $H_{R} \backslash A$ has a perfect matching of type 1 .

Coloring of $K_{t \times s}$ generated by a $s \times t$ matrix. Let $A=\left(a_{i j}\right)_{s \times t}$ be a matrix with distinct elements in each column. The multipartite graph with $t$ partite sets generated by $A$, namely $G=G(A)$, is defined by $V(G)=\left\{v_{j, i} \mid 1 \leq i \leq s, 1 \leq j \leq t\right\}$ and $E(G)=\left\{\left(v_{j, i}, v_{j^{\prime}, i^{\prime}}\right) \mid a_{i j}=a_{i^{\prime} j^{\prime}}\right\}$, where the $t$ partite sets are respectively given by $C_{j}=\left\{v_{j, i} \mid i=1, \ldots, s\right\}$ for $j=1, \ldots, t$.

Given a graph $G=G(A)$ as above, the red and blue two coloring of $K_{t \times s}=H_{R} \oplus H_{B}$ such that $H_{R} \equiv G$ and $H_{B} \equiv G^{c}$ is said to be the two coloring generated by $A$.

Remark 1.1. For $j \geq s+1$, consider a $s \times j$ matrix $A=\left(a_{i, j}\right)$ such that,

- $a_{i, j} \neq a_{p, q}$ whenever $(i \geq j$ and $p \geq q)$ or $(s \geq j>i$ and $s \geq q>p)$,

- if $a_{i, j}=a_{p, q}$, where $i<j$ and $s \geq p>q$, then $i<p$,

- each element appears exactly twice.

Such a matrix $A$ will generates a bad coloring of Type I on $K_{j \times s}$. Given a matrix $A$, satisfying the above conditions, the $s \times(j+2)$ matrix $B=\left(b_{i, j}\right)$ defined by,

$$
B=\left(\begin{array}{cccccccc}
\mathbf{x}_{\mathbf{1}} & a_{1,1} & a_{1,2} & \ldots & a_{1, s} & \ldots & a_{1, j} & \mathbf{x}_{\mathbf{1}} \\
a_{2,1} & \mathbf{x}_{\mathbf{2}} & a_{2,2} & \ldots & a_{2, s} & \ldots & a_{2, j} & \mathbf{x}_{\mathbf{2}} \\
a_{3,1} & a_{3,2} & \mathbf{x}_{\mathbf{3}} & \ldots & a_{3, s} & \ldots & a_{3, j} & \mathbf{x}_{\mathbf{3}} \\
\vdots & \vdots & \vdots & \vdots & \vdots & \vdots & \vdots & \vdots \\
a_{s, 1} & a_{s, 2} & a_{s, 3} & \ldots & \mathbf{x}_{\mathbf{s}} & \ldots & a_{s, j} & \mathbf{x}_{\mathbf{s}}
\end{array}\right)
$$

where $x_{1}, x_{2}, \ldots, x_{s}$ are distinct numbers which do not appear in $A$, will also satisfy the above conditions and hence generate a bad coloring of type I on $K_{(j+2) \times s}$. We leave this remark for the reader to verify.

Furthermore, given a $s \times j$ matrix $A$, which generate a type I coloring on $K_{j \times s}$, one can generate a type II coloring on $K_{(j+1) \times s}$ by adding a new partite set whose vertices are isolated in $H_{R}$. 
Proposition 1.3. $K_{j \times s}$ has a bad coloring of Type 1 or 2 if and only if $j \geq s+1$.

Proof. Suppose $j<s+1$ and $G=H_{R} \oplus H_{B}$ is a bad coloring of Type 1 or 2. Let $A=\left\{a_{1}, \ldots, a_{s}\right\}$ be a partite set with no isolated vertices in $H_{R}$. Then, each $a_{i}$ is incident to a unique partite set $A_{j} \neq A$ in $H_{R}$. Since the coloring is a Type 1 or 2 bad coloring, $A_{i} \neq A_{j}$ for $i \neq j$. This is a contradiction because $j-1<s$. Therefore, if $K_{j \times s}$ has a Type 1 or 2 bad coloring, then $j \geq s+1$.

To prove the converse, consider the following cases.

Case 1: $j=s+1+2 k$ where $k \geq 0$.

When $k=0$, consider the coloring of $K_{(s+1) \times s}$ generated by the $s \times(s+1)$ matrix

$$
A=\left(\begin{array}{cccccc}
s^{2}+1 & a_{1,2} & a_{1,3} & \ldots & a_{1, s} & s^{2}+1 \\
a_{1,2} & s^{2}+2 & a_{2,3} & \ldots & a_{2, s} & s^{2}+2 \\
a_{1,3} & a_{2,3} & s^{2}+3 & \ldots & a_{3, s} & s^{2}+3 \\
\vdots & \vdots & \vdots & \vdots & \vdots & \vdots \\
a_{1, s} & a_{2, s} & a_{3, s} & \ldots & s^{2}+s & s^{2}+s
\end{array}\right),
$$

where $a_{i, j}$ 's are distinct and greater than $s^{2}+s$. Note that, this matrix satisfies the conditions in Remark 1.1. Thus, the coloring generated by $A$ is a bad coloring of type I. Using the part two of the Remark 1.1 and induction, the result follows.

Case 2: $j=s+2+2 k$ where $k \geq 0$.

From the previous case and applying the Remark 1.1 once, we can generate a type II coloring as required.

Proposition 1.4. If $j \geq s+1$, where $s=\left\lceil\frac{n}{j-2}\right\rceil$, then $m_{j}\left(P_{3}, B_{n}\right) \geq\left\lceil\frac{n+1}{j-2}\right\rceil$.

Hence $m_{j}\left(P_{3}, B_{n}\right)=\left\lceil\frac{n+1}{j-2}\right\rceil$.

Proof. We consider two cases.

Case 1: $n \equiv 0 \bmod (j-2)$

From Proposition 1.3, the graph $K_{j \times s}$ has a Type 1 or 2 bad coloring $K_{j \times s}=H_{R} \oplus H_{B}$. Then there is no $P_{3}$ in $H_{R}$ and the largest book $B_{m}$ in $H_{B}$ is such that

$$
m=(j-2) s-1=\left\lceil\frac{n}{j-2}\right\rceil(j-2)-1=n-1 .
$$

Therefore, the graph contains no blue $B_{n}$. Hence

$$
m_{j}\left(P_{3}, B_{n}\right) \geq s+1=\left\lceil\frac{n}{j-2}\right\rceil+1=\left\lceil\frac{n+1}{j-2}\right\rceil .
$$


Case 2: $n \not \equiv 0 \bmod (j-2)$

In this case

$$
\left\lceil\frac{n+1}{j-2}\right\rceil=\left\lceil\frac{n}{j-2}\right\rceil \leq m_{j}\left(P_{3}, B_{n}\right),
$$

where the last inequality follows from Proposition 1.1. The required equality will follow from this together with Proposition 1.2.

Proposition 1.5. If $j<s+1$, where $s=\left\lceil\frac{n}{j-2}\right\rceil$, then $m_{j}\left(P_{3}, B_{n}\right)=\left\lceil\frac{n}{j-2}\right\rceil$.

Proof. Consider a red $P_{3}$-free coloring of $K_{j \times s}=H_{R} \oplus H_{B}$, where $s=\left\lceil\frac{n}{j-2}\right\rceil$. From the Proposition 1.3, this can not be a Type 1 or 2 bad coloring. Then, in $H_{R}$, one of the following conditions should hold.

- There exists a pair of edges $e_{1}=\left(v_{1}, v_{2}\right)$ and $e_{2}=\left(u_{1}, u_{2}\right)$ between two distinct partite sets $A$ and $B$, such that $v_{1}, u_{1} \in A$ and $v_{2}, u_{2} \in B$.

- There exists an isolated vertex $u$ in partite set $X$ and an edge $e=v_{1} v_{2}$ between partite sets $X$ and $Y$.

- There exist two isolated vertices in two distinct partite sets.

In each of these cases, there is a blue book $B_{m}$ where

$$
m=(j-2) s=(j-2)\left\lceil\frac{n}{j-2}\right\rceil \geq n .
$$

Thus, every coloring of $K_{j \times s}$ contains a red $P_{3}$ or a blue $B_{n}$. This together with Proposition 1.1 will give us the required result.

\section{Upper and lower bounds for the size Ramsey numbers paths of size four verses books}

\section{Bad colorings - Type 3 and Type 4}

Definitions of Type 3 and Type 4 bad colorings (red and blue) of $K_{r \times t}\left(=H_{R} \oplus H_{B}\right)$ are given bellow.

- Type 3: $H_{R}$ consists of a disjoint union of three cycles with every pair of three cycles is incident to at least five or six partite sets

- Type 4: $H_{R}$ consists of a partite set, $A$, consisting of only isolated vertices and $H_{R} \backslash A$ has a decomposition of type 3 . 


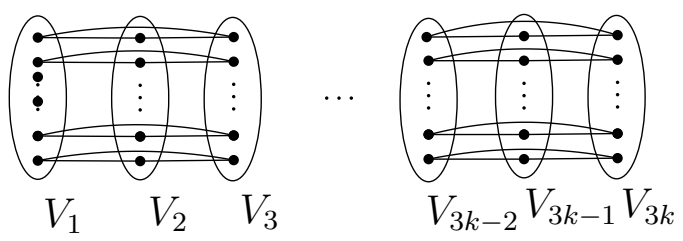

Figure 1. Partitioning $B_{r}$ into 3 disjoint cycles

Proposition 2.1. $m_{j}\left(P_{4}, B_{n}\right) \geq\left\lceil\frac{n+2}{j-2}\right\rceil$ with $j \geq 3$ and $j \neq 5$.

Proof. Case 1: $j \equiv 0 \bmod 3$

Consider the coloring $K_{j \times s}=H_{R} \oplus H_{B}$, where $s=\left\lceil\frac{n+2}{j-2}\right\rceil-1$, such that $B_{R}$ is partitioned in to disjoint 3 cycles as illustrated in the following graph. Note that $V_{1}, V_{2}, \ldots V_{3 k-1}, V_{3 k}$, where $j=3 k$ represent the $j$ partite sets of $K_{j \times s}$.

Then, the graph has no blue $P_{4}$. Moreover, if $B_{m}$ is a blue book contained in the graph then

$$
m \leq(j-2) s-2=\left(\left\lceil\frac{n+2}{j-2}\right\rceil-1\right)(j-2)-2<\left(\frac{n+2}{j-2}\right)(j-2)-2=n .
$$

Therefore, the graph contains no blue $B_{n}$. Hence, $m_{j}\left(P_{4}, B_{n}\right) \geq\left\lceil\frac{n+2}{j-2}\right\rceil$.

Case 2: $j \equiv 1 \bmod 3$

Consider the graph $K_{j \times s}$, where $s=\left\lceil\frac{n+2}{j-2}\right\rceil-1$ such that except for the first partite consisting of isolated vertices of the red graph the remaining $j-1$ partite sets are partitioned in to 3 cycles as done in the case 1 . Then, the graph has no blue $P_{4}$. Moreover, if $B_{m}$ is a blue book contained in the graph then

$$
m \leq(j-2) s-2=\left(\left\lceil\frac{n+2}{j-2}\right\rceil-1\right)(j-2)-2<n .
$$

Therefore, the graph contains no blue $B_{n}$. Hence, $m_{j}\left(P_{4}, B_{n}\right) \geq\left\lceil\frac{n+2}{j-2}\right\rceil$.

Case 3: $j \equiv 2 \bmod 3$

Consider the graph $K_{j \times s}$, where $s=\left\lceil\frac{n+2}{j-2}\right\rceil-1$ such that the vertices are partitioned in to edges and 3 cycles as shown in the following Subcases.

Subcase 1: $s \equiv 0 \bmod 3$

Consider the coloring generated on $K_{j \times s}$, where $s=3 q$, by the matrix given below. 


$\left(\begin{array}{ccccccc}j-1 & j & 1 & 2 & \ldots & j-3 & j-2 \\ j & 1 & 2 & 3 & \ldots & j-2 & j-1 \\ 1 & 2 & 3 & 4 & \ldots & j-1 & j \\ \hline 2 j-1 & 2 j & j+1 & j+2 & \ldots & 2 j-3 & 2 j-2 \\ 2 j & j+1 & j+2 & j+3 & \ldots & 2 j-2 & 2 j-1 \\ j+1 & j+2 & j+3 & j+4 & \ldots & 2 j-1 & 2 j \\ \hline \vdots & \vdots & \vdots & \vdots & \vdots & \vdots & \vdots \\ \hline q j-1 & q j & (q-1) j+1 & (q-1) j+2 & \ldots & 2 j-3 & 2 j-2 \\ q j & (q-1) j+1 & (q-1) j+2 & (q-1) j+3 & \ldots & 2 j-2 & 2 j-1 \\ (q-1) j+1 & (q-1) j+2 & (q-1) j+3 & (q-1) j+4 & \ldots & q j-1 & q j\end{array}\right)$

In this coloring, all the vertices of $B_{R}$ are partitioned in to 3 cycles. If $B_{m}$ is a book contained in $H_{B}$,

$$
m \leq(j-2) s-2=\left(\left\lceil\frac{n+2}{j-2}\right\rceil-1\right)(j-2)-2<n .
$$

Therefore, the graph contains no blue $B_{n}$. Hence, $m_{j}\left(P_{4}, B_{n}\right) \geq\left\lceil\frac{n+2}{j-2}\right\rceil$.

Subcase 2: $s \equiv 1 \bmod 3$

Let $A=\left(a_{i, j}\right)_{3 q \times j}$, where $j=3 r+2(r \neq 1$ as $j \neq 5)$ and $s=3 q+1$, be the matrix defined in subcase 1. Consider the coloring generated on $K_{j \times s}$ by the matrix $B=\left(b_{i, j}\right)_{3 q+1 \times j}$ given below, where $x>\max \left\{a_{i, j}\right\}$.

$$
\left(\begin{array}{ccccccccc}
a_{1,1} & a_{1,2} & a_{1,3} & a_{1,4} & a_{1,5} & \ldots & a_{1, j-2} & a_{1, j-1} & a_{1, j} \\
a_{2,1} & a_{2,2} & a_{2,3} & a_{2,4} & a_{2,5} & \ldots & a_{2, j-2} & a_{2, j-2} & a_{2, j} \\
\vdots & \vdots & \vdots & \vdots & \vdots & \vdots & & & \\
a_{s-1,1} & a_{s-1,2} & a_{s-1,3} & a_{s-1,4} & a_{s-1,5} & \ldots & a_{s-1, j-2} & a_{s-1, j-1} & a_{s-1, j} \\
x & x+1 & x+1 & x+1 & x & \ldots & x+r & x+r & x+r
\end{array}\right)
$$

In this coloring, all the vertices of $B_{R}$ are partitioned in to 3 cycles, except for the two vertices $v_{1, s}$ and $v_{5, s}$, incident to the edge $\left(v_{1, s}, v_{5, s}\right)$, corresponding to the entries $b_{s, 1}=b_{s, 5}=x$.

If $B_{m}$ is a book in $H_{B}$. Then,

$$
m \leq(j-2) s-2=\left(\left\lceil\frac{n+2}{j-2}\right\rceil-1\right)(j-2)-2<n .
$$

Therefore, the graph contains no blue $B_{n}$. Hence, $m_{j}\left(P_{4}, B_{n}\right) \geq\left\lceil\frac{n+2}{j-2}\right\rceil$.

Subcase 3: $s \equiv 2 \bmod 3$

Let $A=\left(a_{i, j}\right)_{3 q \times j}$, where $j=3 r+2(r \neq 1$ as $j \neq 5)$ and $s=3 q+2$, be the matrix defined in subcase 1. Consider the coloring generated on $K_{j \times s}$ by the matrix $C=\left(b_{i, j}\right)_{(3 q+2) \times j}$ given below, where $x>\max \left\{a_{i, j}\right\}$ and $y>x+r$. 


$$
\left(\begin{array}{ccccccccc}
a_{1,1} & a_{1,2} & a_{1,3} & a_{1,4} & a_{1,5} & a_{1,6} & \ldots & a_{1, j-1} & a_{1, j} \\
a_{2,1} & a_{2,2} & a_{2,3} & a_{2,4} & a_{2,5} & a_{2,6} & \ldots & a_{2, j-2} & a_{2, j} \\
\vdots & \vdots & \vdots & \vdots & \vdots & \vdots & & & \\
a_{s-1,1} & a_{s-1,2} & a_{s-1,3} & a_{s-1,4} & a_{s-1,5} & a_{s-1,6} & \ldots & a_{s-1, j-1} & a_{s-1, j} \\
x & x+1 & x+1 & x+1 & x & x+2 & \ldots & x+r & x+r \\
y+r & y & y+1 & y+1 & y+1 & y & \ldots & y+r & y+r
\end{array}\right)
$$

In this coloring, all the vertices of $C_{R}$ are partitioned in to 3 cycles, except for the following two cases.

- Two vertices $v_{1, s-1}$ and $v_{4, s-1}$, incident to the edge $\left(v_{1, s-1}, v_{4, s-1}\right)$, corresponding to the entries $c_{s-1,1}=b_{s-1,4}=x$.

- Two vertices $v_{2, s}$ and $v_{5, s}$, incident to the edge $\left(v_{2, s}, v_{5, s}\right)$, corresponding to the entries $c_{s, 2}=$ $c_{s, 5}=y$.

If $B_{m}$ is a book in $H_{B}$. Then,

$$
m \leq(j-2) s-2=\left(\left\lceil\frac{n+2}{j-2}\right\rceil-1\right)(j-2)-2<n
$$

Therefore, the graph contains no blue $B_{n}$. Hence, $m_{j}\left(P_{4}, B_{n}\right) \geq\left\lceil\frac{n+2}{j-2}\right\rceil$.

We omit the proof of the following proposition since it is similar to the proof of Proposition 1.3.

Proposition 2.2. If $j<2 s+1$ then $K_{j \times s}$ has no Type 3 or 4 bad coloring.

Theorem 2.1. Let $s=\left\lceil\frac{n+2}{j-2}\right\rceil$. If $j \neq 5$ and $j<2 s+1$ or $n+2 \not \equiv 0 \bmod (j-2)$, then $m_{j}\left(P_{4}, B_{n}\right)=\left\lceil\frac{n+2}{j-2}\right\rceil$.

Proof. We consider two cases.

Case 1: $j<2 s+1$

Consider a red $P_{4}$-free red/blue coloring of $K_{j \times s}=H_{R} \oplus H_{B}$, where $s=\left\lceil\frac{n+2}{j-2}\right\rceil$. From Proposition 2.2, this cannot be a Type 3 or 4 bad coloring. Therefore, in $H_{B}$, there exists a pair of 
vertices $v_{1}$ and $v_{2}$ belonging to two distinct partite sets $A$ and $B$, such that $v_{1}$ and $v_{2}$ are adjacent to at most two vertices in $H_{R} \backslash\{A \cup B\}$. This forces a book $B_{m}$ where

$$
m=(j-2) s-2=(j-2)\left\lceil\frac{n+2}{j-2}\right\rceil-2 \geq n
$$

Thus, every coloring of $K_{j \times s}$ contains a red $P_{4}$ or a blue $B_{n}$. Hence the result.

Case 2: $n+2 \not \equiv 0 \bmod (j-2)$

From Proposition 2.2, we get $\left\lceil\frac{n+2}{j-2}\right\rceil \leq m_{j}\left(P_{4}, B_{n}\right) \leq\left\lceil\frac{n+3}{j-2}\right\rceil$. But we know that, $\left\lceil\frac{n+2}{j-2}\right\rceil=$ $\left\lceil\frac{n+3}{j-2}\right\rceil$, because $n+2 \not \equiv 0 \bmod (j-2)$. Hence the result.

\section{References}

[1] S. A. Burr, P. Erdös, R. J. Faudree, R. J. Gould, M. S. Jacobson, C. C. Rousseau, and R. H. Schelp, Goodness of trees for generalized books, Graphs Combin. 3 (1987), 1-6.

[2] A.P. Burger and J.H. van Vuuren, Ramsey numbers in Complete Balanced Multipartite Graphs. Part II: Size Numbers, Discrete Math. 283 (2004), 45-49.

[3] P. Erdös, J. Faudree, C. C. Rousseau, and J. Sheehan, The size Ramsey number, Period Math. Hungary (1978), 145-161.

[4] R. J. Faudree, C. C. Rousseau, and J. Sheehan, Cycle-book Ramsey numbers, Ars Combinatorica 31 (1991), 239-248.

[5] S.P. Radziszowski, Small Ramsey numbers, Electronic Journal of Combinatorics 14 (2014), DS1.

[6] C. C. Rousseau and J. Sheehan, On Ramsey numbers for books, Journal of Graph Theory 2 (1978), 77-87.

[7] Syafrizal Sy, E.T. Baskoro and S. Uttunggadewa, The size multipartite Ramsey number for paths, Journal Combin. Math. Combin. Comput. 55 (2005), 103-107.

[8] Syafrizal Sy, E.T. Baskoro, S. Uttunggadewa and H. Assiyatun, Path-path size multipartite Ramsey numbers, Journal Combin. Math. Combin. Comput. 71 (2009), 265-271.

[9] Syafrizal Sy, and E.T. Baskoro, Lower bounds of the size multipartite Ramsey numbers, The 5th Mathematics, AIP Conf. Proc. 1450 (2012), 259-261.

[10] Y. Li and C. C. Rousseau, n Book-Complete Graph Ramsey Numbers, Journal of combinatorial theory. Series B 68 (1996), 36-44. 